\title{
Who is at risk? Gendered psychological correlates in Italian students' sexual risk profiles
}

\author{
Elena Pirani ${ }^{1^{*}}$ (D) and Camilla Matera ${ }^{2}$
}

\author{
*Correspondence: elena.pirani@ \\ unifi.it \\ ${ }^{1}$ Dipartimento di Statistica, \\ Informatica, Applicazioni - DiSIA, \\ University of Florence, Viale \\ Morgagni 59, 50134 Firenze, Italy \\ Full list of author information is \\ available at the end of the article
}

\begin{abstract}
Recent research has shown that sexual risk behaviours are rising substantially among Italian youth, but this evidence is still undervalued in public discussion. Adopting a multidimensional perspective for sexual risk and addressing gender differences, the present study investigates if and how some individuals' psychological characteristicsnamely body satisfaction, self-esteem and body sensation-seeking - are associated with unsafe and risky sexual behaviours of Italian emerging adults. We exploited the survey SELFY_Sexual and Emotional LiFe of Youths_conducted in 2017 in Italy. Several key points may be highlighted from our study. First, we identify two clusters characterized by high sexual risk-taking behaviours, which include one third of our sample, a nonnegligible proportion. Second, we prove that women are generally less inclined to adopt risk-taking behaviours in sexual life. Third, our findings show that body satisfaction, self-esteem and sensation seeking are differently linked to sexual risk taking among women and men. Body satisfaction is associated with a higher probability of adopting risky and promiscuous sexual behaviours among men, while the relationship is not significant among women. Sensation seeking shows a certain relevance in defining sexual risk profiles, especially for men. Overall, our research suggests that body image and self-worth could be relevant areas of intervention for sexual health programs targeting emerging Italian adults. Notably, communication and education strategies on these issues should be differentiated for women and men.
\end{abstract}

Keywords: Sexual behaviour, Risk taking, Body image, Self-esteem, Sensation seeking, Italy

\section{Introduction}

Researchers define risky sexual behaviour as comprising sexual activities that expose individuals to the risk of contracting sexually transmitted infections (STIs), with significant consequences to their health (Chawla \& Sarkar, 2019; Italian National Institute of Health-ISS, 2018a, 2018b; Satterwhite et al., 2013). STIs constitute a group of infectious diseases that affect millions of people every year (European Centre for Disease Prevention and Control-ECDC, 2017). In many Western countries, STIs are still a public health concern: every day, there are more than 1 million new STI cases among people aged 15-49 years (Rowley et al., 2019), and approximately half of newly diagnosed STIs occur among adolescents and young adults between the ages of 15 and 24

(c) The Author(s). 2020 Open Access This article is licensed under a Creative Commons Attribution 4.0 International License, which permits use, sharing, adaptation, distribution and reproduction in any medium or format, as long as you give appropriate credit to the original author(s) and the source, provide a link to the Creative Commons licence, and indicate if changes were made. The images or other third party material in this article are included in the article's Creative Commons licence, unless indicated otherwise in a credit line to the material. If material is not included in the article's Creative Commons licence and your intended use is not permitted by statutory regulation or exceeds the permitted use, you will need to obtain permission directly from the copyright holder. To view a copy of this licence, visit http://creativecommons.org/licenses/by/4.0/. 
(Italian National Institute of Health, 2018a; Satterwhite et al., 2013). Risky sexual behaviour is the leading cause of STIs, including, but not limited to, HIV. According to the ISS, year-by-year relevant growth is recorded for various types of STIs in Italy (Italian National Institute of Health, 2018a, 2018b): in 2017, about 11 new cases of STIs per 100,000 inhabitants were diagnosed, and the HIV infection incidence was 5.8 new cases per 100,000 inhabitants, in line with the European average. Among the most widespread infections, the incidence of syphilis increased by $70 \%$ in 2016 compared to the year before; the number of new chlamydia infections, which mainly affect young women, doubled compared to 2010; and the cases of condyloma, the most reported STI, tripled in the last 10 years. Notably, the trends are intensifying dramatically, especially among younger people (Italian National Institute of Health, 2018a).

This evidence raises concerns about the practice of unsafe sex among young adults, many of whom engage in risky sexual behaviours, although with some variation across different countries (e.g., De Looze et al., 2019). In Italy, some recent research has shown that sexual risk behaviours are rising substantially among young adults. For instance, a survey conducted in 2017 on a sample of about 8000 Italian university students (Dalla Zuanna et al., 2019) revealed that the $20 \%$ of them had their first sexual intercourse without any contraception or only relying on withdrawal; moreover, the survey's results demonstrated that a substantial percentage of students voluntarily engaged in unprotected sexual intercourse with occasional partners (29\% among men, $12 \%$ among women). Similarly, through a Web survey conducted in 2015 on about 4600 students attending Sapienza University of Rome, Liuccio et al. (2019) found that participants' risk perceptions for sexual health were low or medium; only a third of the sample used condoms regularly, with no significant differences between female and male students. Even when risk perception was high, this perception did not result in safer sexual behaviours. Identifying the factors associated with young adults' sexually risky behaviours is thus pivotal for programs and interventions seeking to promote safe and health-preserving behaviours.

Traditional socio-demographic characteristics, such as educational level or educational aspirations, and family factors, such as parental education or lack of communication about contraception (e.g., Brooks-Gunn and Furstenberg, 1989; De Looze et al., 2019; Luster and Small, 1994), have been investigated and identified as important correlates of sexual risk taking among emerging adults. These studies suffer from several limitations, however. First, the majority of studies examining sexual risk considered only one single behaviour at a time, such as a high number of sexual partners, inconsistent or rare condom use, or sexual intercourse with casual partners (e.g., Durbin et al., 1993; Whitaker and Miller, 2000). When multiple behaviours were considered, they were examined in separate analyses (e.g., Secor-Tuner et al., 2015). Second, these studies overlooked the role of psychological characteristics. Among them, some might be especially important, such as self-perceptions-including body satisfaction (e.g., Blashill and Safren, 2015; Gillen et al., 2006; Merianos et al., 2013) and self-esteem (e.g., Ethier et al., 2006; McGee and Williams 2000)—and personality traits-including impulsivity and sensation seeking (e.g., Arnold et al., 2002; Gullette and Lyons, 2005; McCoul and Haslam, 2001). Third, the majority of these studies referred to specific and selected subgroups of the population, like sexual minorities (e.g., Gholizadeh et al., 2018; Salazar et al., 2005; Spitalnick et al., 2007; Wingood et al., 2002). Finally, the 
studies often failed to account for the role of gender in shaping the effects of psychological variables (McGee and Williams, 2000; Potard et al., 2019).

This study adds to the literature by examining the sexual risk profiles of Italian students from new angles: (1) adopting a multidimensional perspective for sexual risk to define specific sexual risk profiles; (2) scrutinising the association between psychological factors-namely, body satisfaction, self-esteem and sexual sensation seeking-and the likelihood of classification into various profiles of risky behaviour, net of traditional socio-demographic and economic correlates; and (3) focusing on gender differences. Further, we used a new, original dataset with representative information of Italian university students enrolled in economics and statistics. Emerging adulthood, which encompasses late adolescence and early adulthood (Arnett, 2000), is likely to be a period during which exploration of sexuality is heightened, with potential consequences including risky sexual behaviours. Many young adults in this age range leave their homes so that they are no longer under the direct supervision of their parents, and this particular condition provides them with higher freedom to engage in sexual behaviours, which enhances the likelihood of risk-taking behavioural patterns.

We proceed as follows. In the "Background" section, we present the multidimensional approach followed in our study for risky sexual behaviour, and we then critically review results concerning the role of body satisfaction, self-esteem and sexual sensation seeking. The section ends with a description of our research hypotheses. In the "Data and method" section, we describe our analytical strategy. The "Results" section presents our findings, both in terms of risky sexual behaviour profiles and the psychological factors that we proved were linked to these profiles. We conclude with a discussion that places our results in the literature of psychological correlates of sexual risk and outlines practical implications for prevention/intervention programs about sexual health in Italy.

\section{Background}

\section{Risky sexual behaviour: a multidimensional approach}

To capture the complexity associated with the sexual experience, a recent line of research has demonstrated that modelling a sexual risk behaviour through a multidimensional approach is more adequate than focusing on single behaviours at once (e.g., Beadnell et al., 2005; Potard et al., 2019; Vasilenko et al., 2015). Different patterns or profiles of sexual risk, covering a large variety of behaviours, should be simultaneously considered to have a complete and meaningful understanding of this issue. Indeed, a single behaviour may be risky not in isolation but rather when it appears in combination with other behaviours (Vasilenko et al., 2015), indicating that engaging in multiple risk behaviours at the same time may enhance one's potential vulnerability to STIs. For instance, for an individual who avoids the use of condoms, the risk of contracting STIs increases enormously if this person has sexual intercourse with casual partners, betrays the partners, or engages in other risky behaviours. However, foregoing the use of condoms may have a different meaning for young people in stable relationships; in these cases, sexual behaviours could be better characterised by considering if other measures of protection are chosen.

The multidimensional approach has proved to be useful for Vasilenko et al. (2015), who grouped adolescents into various classes according to different aspects of their 
sexual behaviours. Due to the longitudinal nature of their data, the authors found that different sexual behaviour patterns predicted young adult STI outcomes, especially for men. Similarly, Potard et al. (2019) defined three different profiles for male and female university students, showing that some domains of self-perception related to physical ability, such as coordination, were related to sexual risk among both women and men. Conversely, appearance-related concerns were found to be related to high-risk sexual behaviours only among women. Overall, the multidimensional approach might offer additional information about behavioural risk patterns, which could be profitably exploited in the development of interventions (Beadnell et al., 2005).

\section{Psychological correlates of risky sexual behaviour}

Risky sexual behaviours may be considered the result of a number of psychological determinants, which range from self-perceptions to personality traits. In particular, personal vulnerabilities to risky behaviours might be reduced by the presence of individual protective factors, such as body satisfaction and self-esteem; otherwise, they might be enhanced by the presence of personality factors, such as sexual sensation seeking. Empirical findings on these issues are still inconsistent, and, most interesting, the protective or enhancing role of these factors with respect to risky sexual behaviours has been found to differ between young men and young women. In the following, we review previous studies on these issues.

\section{Body satisfaction}

With respect to appearance-related concerns, previous research has shown that body dissatisfaction, a central perceptive component of body image (Cash and Pruzinsky, 2002; Thompson, 1990), can enhance risky sexual behaviours (Blashill and Safren, 2015). Indeed, satisfaction with one's appearance and sexuality are physically oriented domains of the self; sexuality involves physical intimacy, which exposes bodies for exploration and judgment by partners (Gillen et al., 2006). These authors found that the link between body satisfaction and risk-taking behaviours differed according to gender. Among young women, positive views of appearance seemed to be a protective factor. It is well established that body dissatisfaction can contribute to lower condom use selfefficacy (Blashill and Safren, 2015), which can decrease intended condom use among girls and women (Matera, 2014) and enhance risky sexual behaviours (Eisenberg et al., 2005; Gillen et al., 2006; Schooler et al., 2005; Wingood et al., 2002). Body appreciation might be linked to increased female empowerment in situations in which the body is displayed, and this correlation might also correspond to higher self-efficacy in negotiating condom use (Gillen et al., 2006).

In men, the relationship between body dissatisfaction and risky sexual behaviours has been examined mostly among sexual minorities (Brady et al., 2019; Gholizadeh et al., 2018). Among heterosexual men, the empirical evidence is inconsistent (Gillen et al., 2006; Merianos et al., 2013; Schooler and Ward, 2006). According to Gillen et al. (2006), positive views of one's body and an orientation towards appearance enhanced men's likelihood of engaging in risky sexual behaviour. Among them, greater body satisfaction could correspond to higher confidence in one's masculinity, which is stereotypically linked to sexual freedom and power. In contrast, Schooler and Ward (2006) 
found that men who were satisfied with their body reported less risky sexual behaviour compared to men who were not satisfied. These authors argued that the embarrassment, shame and discomfort that might be associated with one's body might lead men into being less assertive in their sexual decisions and activity. This might be linked to less safe sexual behaviour, as is true for women. In a recent study in the Italian context, Nerini and Matera (2020) found that men's beliefs about control over their appearance were negatively associated with intended condom use; the more men believed they could control their appearance, the less they intended to use a condom during sexual intercourse with casual partners. Nevertheless, a higher tendency to monitor one's body was related to a greater probability of using some condom negotiation strategies to obtain protected sex.

\section{Self-esteem}

Both body image and engagement in safe sexual behaviours are closely linked to selfesteem, which refers to how good, worthy or competent individuals generally view themselves (Rosenberg et al., 1995). Increasing evidence suggests that low self-esteem has a negative impact on a variety of health-related, risky behaviours, such as poor nutritional habits (e.g., Timmerman and Acton, 2001). Conversely, high self-esteem positively enhances behaviours such as physical activity and exercise (e.g., McAuley et al., 2000).

Research on the relationship between self-esteem and sexual risk behaviour has produced conflicting results. Some studies found a significant negative relationship between self-esteem and risky sexual behaviour (Boden and Horwood, 2006; Enejoh et al., 2016; Ethier et al., 2006; McNair et al., 1998; Olaoye and Agbede, 2019; Preston et al., 2004; Salazar et al., 2005; Sterk et al., 2004), others highlighted a positive association between these two variables (Kim et al., 2018) and additional studies failed to demonstrate a significant relationship between self-esteem and risky sexual behaviour (Goodson et al., 2006; McGee and Williams, 2000).

In most of these studies, gender and self-esteem have been considered independent predictors of risky sexual behaviours. An exception appears in the study by Tevendale et al. (2009) on homeless youth, a highly selected group: the authors showed that lower self-esteem was associated with a higher likelihood of unprotected sex among girls, but this relationship was found to be not significant among boys. These findings suggest that, as in the case of body satisfaction, gender might moderate the association between self-esteem and risky sexual behaviours.

\section{Sensation seeking}

The role of personality characteristics such as sensation seeking in risky sexual behaviour has been investigated as well. Sensation seeking describes one's propensity for exciting, optimal and novel levels of stimulation (Kalichman et al., 1994), and sensation-seeking individuals tend to engage in behaviours that increase the amount of stimulation they experience in order to seek arousal. Sexual intercourse, which is typically accompanied by physical excitement and high emotions (Matera et al., 2018; Norton et al., 2005), represents an emblematic way to obtain this kind of stimulation. Youth with higher levels of sexual sensation seeking might be more interested in having new sexual experiences than 
in seeking emotional connection with current partners, which could expose them to higher risk (Ritchwood et al., 2015).

Indeed, sexual sensation seeking has been proved to be an important factor in risky sexual behaviour, including frequent intercourse, more sexual partners and reduced condom use (Gullette and Lyons, 2005; McCoul and Haslam, 2001; Spitalnick et al., 2007; Voisin et al., 2013). This association has been examined mostly among gay and bisexual men, whereas the role of sensation seeking in determining risky sexual behaviour among heterosexual youth is less explored. The few studies conducted on English and American students showed that men scored higher than women in sensation seeking and were likely to have more sexual partners (Arnold et al., 2002; Rolison and Scherman, 2002). Even though sensation seeking significantly contributed to risk-taking behaviours of youth regardless of gender, men with higher levels of sensation seeking were significantly more likely than women to participate in risk-taking behaviours related to HIV transmission and were less likely to have a steady sex partner (Gullette and Lyons, 2005).

\section{Research hypotheses}

Are sexual risk-taking patterns detectable for Italian youth? Are sexual risk behaviours linked to psychological individual characteristics such as body satisfaction, self-esteem and sensation seeking? Are these associations shaped by gender?

First of all, we expect that different risk profiles-and not only a high-low risk dichotomisation-can be identified among Italian university students. Previous research adopting a multidimensional perspective indicated that risky sexual behaviours interact one another (e.g., Vasilenko et al., 2015), resulting in various risk patterns.

In addition, regarding the link between psychological factors and sexually risky behaviours, we predict that promiscuous and risk-taking behaviours are more likely to characterise young men displaying higher levels of body satisfaction and self-esteem. A man who likes himself and his body is more likely to feel that he is conforming to a widespread ideal of masculinity, which in turn might enhance his perception of being powerful. Such a feeling of invulnerability might thus contribute to a man's belief that he does not have to protect himself (Gillen et al., 2006). Conversely, we expect that, for young women, body satisfaction and self-esteem represent protective factors that prevent engagement in risky sexual behaviours. If a woman does not like her body and does not value herself, she will probably feel embarrassed and unable to ask a man to use protection during sex. This gendered association may especially matter in the Italian context, where masculinity is still stereotypically linked to perceived power (Matera et al., 2019) and women are typically socialised to believe that they are weak and fragile (Biemmi, 2015).

Finally, we advance that sexual risk-taking profiles will be prevalent among individuals who like to feel and live their body intensely (that is, with high sexual sensationseeking levels), especially among men. Indeed, seeking sensations and emotions could have different meanings based on gender; unlike among women, men's main need during sexual intercourse could be the achievement of pleasure, which is ostensibly heightened when a condom is not used (Randolph et al., 2007). As a consequence, the relation between sensation seeking and unprotected sex is predicted to be stronger for men than for women. 


\section{Data and method}

\section{Data and analytical strategy}

This study was based on the survey on Sexual and Emotional LiFe of Youths-SELFY. This survey was conducted in 2017 in 28 Italian universities with the aim of drawing an updated picture of sexual and emotional opinions and behaviours among Italian university students. For more details on the survey methodology and the characteristics of the respondents, see Dalla Zuanna et al. (2019). The respondents-7840 individuals, 52.4\% men-were all attending undergraduate courses in economics and statistics in Italian public universities.

Our empirical analysis comprised two steps. First, we performed a cluster analysis to aggregate individuals into homogeneous groups according to their safe and risky sexual behaviours. Second, we estimated a multinomial logistic regression model to appreciate the association between the identified risk profiles and three psychological characteristics: body satisfaction, self-esteem and sensation seeking. In line with our focus on gender differences, we augmented our statistical model with an interaction between psychological factors and gender.

In this analysis, we focused on individuals who already had sexual intercourse at least once in their lives, and these individuals constituted $80 \%$ of the original sample size. Due to some missing values for the variables of sexual risk behaviours considered (almost all ranging from 0.9 to $2.9 \%$; the number of partners was not available for $11.3 \%$ of the target sample), the analyses focused on 5207 young adults aged 18 to 26 years old.

\section{Profiling individuals: cluster analysis}

We used cluster analysis to define various profiles of sexual risk behaviours. Cluster analysis (e.g., Everitt, 1974) is an exploratory statistical technique aimed at grouping observations (individuals in this case) according to a set of characteristics. Respondents in the same group are more similar to each other than they are to those in the other groups. Because our aim was to define sexual risk-taking profiles following a multidimensional approach, the variables used to group individuals referred to several sex behaviours included in the questionnaire, concerning both the first sexual experience that participants had and their sexual lives at the time of the interview.

First, we considered the age at first intercourse and the number of sexual partners the individuals had in their lives. The former was aggregated into three classes in an attempt to form equal sample sizes (16 or less; 17-18; 19 or greater). The latter was categorised into $1 ; 2 ; 3 ; 4 ; 5$; and 6 or more; we preferred to maintain a certain level of detail to better analyse differences among participants according to their numbers of sexual partners. Because our sample included young people aged 18-26, the total number of partners they declared was generally low: 70\% of the sample had from 1 to 3 sexual partners (50\% had only 1 or 2 ).

The use of contraception and the type of contraception are crucial when studying risk in sexual behaviour. We thus considered different variables. We considered contraception used in the first and in the most recent sexual intercourse (nothing; rhythm method; withdrawal; condom, pill, or patch; ring or IUD; don't remember). We considered also if the respondents had discussed sexual protection with their partners before their first intercourse (no; yes; don't remember) and whether the respondents had sexual 
intercourse without a condom with casual partners (never; sometimes; often; very often). We then included a variable indicating the type of relationship with the first sexual partner (the stable partner of that period; a friend/acquaintance; an unknown person). Sexual lifestyle choices were also measured; respondents were asked if they had ever had sexual intercourse without being in love with the partner (never; sometimes; often; very often), if the respondents had ever had sex with people who were not their current partners while in relationships (never; sometimes; often; very often), and if they had ever paid to have sex (no; yes). Finally, we considered the frequency of sexual intercourse in the last 3 months (never; 1 time per month or rarely; 2-3 times per month; several times per week). The complete wording of the questions considered is reported in Table 1.

Because the variables we used to group individuals were ordinal or categorical (the quantitative variables used were aggregated in classes), we opted for a partitionclustering method (i.e., the $k$-median cluster analysis) using the Manhattan distance (Jain and Dubes, 1988; Strauss and von Maltitz, 2017). We preliminarily tried various specifications (e.g., $k$-mean, also considering the city block distance) and other methods (e.g., hierarchical clustering). Results were not substantially different in the various specifications, proving the robustness of our results.

The final clustering grouped individuals into five sexual risk profiles (Table 1). This clusterisation was able to substantially differentiate individuals according to their attitudes and behaviours toward sex and sexual intercourse, preserving the sample size of each group. A higher number of groups could have been possible; nevertheless, we think that groups too small in size would undermine the significance of subsequent analysis without improving our findings from a substantive point of view (e.g., Kaufman and Rousseeuw, 2005).

\section{Psychological characteristics and risk profiles: multinomial analysis}

The second step of our analysis consisted of assessing the association between the risktaking profiles identified in the first step and some individual psychological characteristics. We used a multinomial logistic regression model in which the response variable was the categorisation into the five clusters. Our final model specification included the following three key explanatory variables referring to the individuals' psychological sphere.

First, according to the previous literature, we considered individuals' body satisfaction to capture physical self-perception, a factor considered central for general self-esteem during adolescence and young adulthood (e.g., Kiviruusu et al., 2016; Stefanile et al., 2009). We used the question asking if, at the time of interview, respondents were satisfied with their physical appearance. For model parsimony, the original response categories were aggregated into not at all or little satisfied, rather satisfied and satisfied. Second, another aspect linked to both self-identity development and interpersonal relationships is self-esteem. This variable has been operationalised according to the level of agreement with the statement "I feel insecure and inadequate; I can't face life situations"; the original responses have been aggregated considering a three-category variable differentiating among disagree (completely or rather), indifference or agree (completely or rather). Third, as discussed above, sensation-seeking seems to lead to intense experiences within sexual relationships. We focused on sexual body sensation seeking, partially due to data availability constraints. This aspect has been proxied 
Table 1 Sexual risk profiles: cluster size and distribution of the variables describing various sexualrelated behaviours by the 5 sexual risk profiles identified through the cluster analysis

\begin{tabular}{|c|c|c|c|c|c|c|}
\hline & $\begin{array}{l}\# 1 \text {. Promiscuous, } \\
\text { high risk }\end{array}$ & $\begin{array}{l}\text { \#2. Risk- } \\
\text { taking }\end{array}$ & $\begin{array}{l}\text { \#3. Few } \\
\text { partners, } \\
\text { low risk }\end{array}$ & $\begin{array}{l}\text { \#4. Monogamous, } \\
\text { medium risk }\end{array}$ & $\begin{array}{l}\text { \#5. Low } \\
\text { sexual } \\
\text { practice }\end{array}$ & Total \\
\hline \multicolumn{7}{|l|}{ Cluster size } \\
\hline abs. val. & 959 & 783 & 2071 & 471 & 923 & 5207 \\
\hline$\%$ val. & 18.4 & 15.0 & 39.8 & 9.1 & 17.7 & 100.0 \\
\hline \multicolumn{7}{|c|}{ Age at first intercourse } \\
\hline$\leq 16$ & 68.4 & 22.6 & 21.5 & 77.9 & 23.2 & 35.7 \\
\hline $17 / 18$ & 25.7 & 56.3 & 45.3 & 17.8 & 44.6 & 40.8 \\
\hline$\geq 19$ & 5.9 & 21.1 & 33.2 & 4.3 & 32.2 & 23.6 \\
\hline \multicolumn{7}{|c|}{ Number of sexual partners } \\
\hline 1 & 0.0 & 0.9 & 69.7 & 0.0 & 52.9 & 37.2 \\
\hline 2 & 0.3 & 20.4 & 23.8 & 11.5 & 32.8 & 19.5 \\
\hline 3 & 1.2 & 41.9 & 5.7 & 34.6 & 9.4 & 13.6 \\
\hline 4 & 4.5 & 24.5 & 0.7 & 27.8 & 3.0 & 7.8 \\
\hline 5 & 13.5 & 8.8 & 0.1 & 17.4 & 1.1 & 5.6 \\
\hline$\geq 6$ & 80.6 & 3.5 & 0.0 & 8.7 & 0.8 & 16.3 \\
\hline \multicolumn{7}{|c|}{ Contraception at first intercourse } \\
\hline Nothing & 12.8 & 13.0 & 5.6 & 7.9 & 10.9 & 9.2 \\
\hline Rhythm method & 0.1 & 0.8 & 0.9 & 0.6 & 1.1 & 0.7 \\
\hline Withdrawal & 11.8 & 11.4 & 7.6 & 11.0 & 6.8 & 9.1 \\
\hline Condom & 66.1 & 67.1 & 77.7 & 67.9 & 74.4 & 72.5 \\
\hline Pill or patch & 7.3 & 6.5 & 7.4 & 10.8 & 5.7 & 7.3 \\
\hline Ring or IUD & 0.3 & 0.4 & 0.3 & 0.9 & 0.0 & 0.3 \\
\hline Don't remember & 1.6 & 0.9 & 0.5 & 0.9 & 1.0 & 0.9 \\
\hline
\end{tabular}

Discussed contraception with a partner before first intercourse

$\begin{array}{lllllll}\text { No } & 49.3 & 40.1 & 16.5 & 26.1 & 38.7 & 30.9 \\ \text { Yes } & 33.6 & 40.9 & 71.3 & 53.3 & 46.6 & 53.8 \\ \text { Don't remember } & 17.1 & 19.0 & 12.3 & 20.6 & 14.7 & 15.4 \\ \text { Partner at first intercourse } & & & & & & \\ \text { The partner of that period } & 65.7 & 45.9 & 96.3 & 94.3 & 82.6 & 80.5 \\ \text { A friend/acquaintance } & 26.9 & 45.0 & 3.5 & 5.3 & 14.4 & 16.1 \\ \text { An unknown person } & 7.4 & 9.2 & 0.2 & 0.4 & 3.0 & 3.4\end{array}$

Ever paid for sex

$\begin{array}{lllllll}\text { No } & 80.5 & 90.8 & 99.1 & 97.2 & 97.2 & 93.9 \\ \text { Yes } & 19.5 & 9.2 & 0.9 & 2.8 & 2.8 & 6.1\end{array}$

Sexual intercourse without being in love with the partner

$\begin{array}{lllllll}\text { Never } & 9.2 & 13.4 & 93.9 & 67.1 & 77.4 & 60.8 \\ \text { Sometimes } & 63.4 & 74.8 & 5.8 & 31.6 & 18.9 & 31.4 \\ \text { Often } & 27.4 & 11.8 & 0.3 & 1.3 & 3.8 & 7.7 \\ \text { Very often } & 0.0 & 0.0 & 0.0 & 0.0 & 0.0 & 0.0\end{array}$

Ever betrayed the partner

$\begin{array}{lllllll}\text { Never } & 38.0 & 81.9 & 96.0 & 79.6 & 95.2 & 81.6 \\ \text { Sometimes } & 51.8 & 17.1 & 3.8 & 19.1 & 4.6 & 16.2 \\ \text { Often } & 7.8 & 0.8 & 0.2 & 1.3 & 0.1 & 1.8 \\ \text { Very often } & 2.4 & 0.3 & 0.0 & 0.0 & 0.1 & 0.5\end{array}$


Table 1 Sexual risk profiles: cluster size and distribution of the variables describing various sexualrelated behaviours by the 5 sexual risk profiles identified through the cluster analysis (Continued)

\begin{tabular}{|c|c|c|c|c|c|c|}
\hline & $\begin{array}{l}\text { \#1. Promiscuous, } \\
\text { high risk }\end{array}$ & $\begin{array}{l}\text { \#2. Risk- } \\
\text { taking }\end{array}$ & $\begin{array}{l}\text { \#3. Few } \\
\text { partners, } \\
\text { low risk }\end{array}$ & $\begin{array}{l}\text { \#4. Monogamous, } \\
\text { medium risk }\end{array}$ & $\begin{array}{l}\text { \#5. Low } \\
\text { sexual } \\
\text { practice }\end{array}$ & Total \\
\hline \multicolumn{7}{|c|}{ Sexual intercourse with a non-stable partner without a condom } \\
\hline Never & 25.7 & 37.7 & 91.4 & 47.6 & 82.1 & 65.6 \\
\hline Sometimes & 49.3 & 50.2 & 5.1 & 39.5 & 13.5 & 24.6 \\
\hline Often & 17.1 & 7.7 & 2.1 & 8.5 & 3.0 & 6.5 \\
\hline Very often & 7.9 & 4.5 & 1.4 & 4.5 & 1.3 & 3.3 \\
\hline \multicolumn{7}{|c|}{ Contraception at last intercourse } \\
\hline Nothing & 17.8 & 16.5 & 7.0 & 8.5 & 9.9 & 11.0 \\
\hline Rhythm method & 1.6 & 1.0 & 1.5 & 0.9 & 0.8 & 1.3 \\
\hline Withdrawal & 19.0 & 13.5 & 11.5 & 9.1 & 8.7 & 12.5 \\
\hline Condom & 44.5 & 56.3 & 58.3 & 22.3 & 69.7 & 54.2 \\
\hline Pill or patch & 14.9 & 11.2 & 19.9 & 52.7 & 9.6 & 18.8 \\
\hline Ring or IUD & 1.7 & 0.9 & 1.5 & 5.7 & 0.4 & 1.6 \\
\hline Don't remember & 0.5 & 0.5 & 0.3 & 0.9 & 1.0 & 0.6 \\
\hline \multicolumn{7}{|c|}{ Frequency of sexual intercourse in the last 3 months } \\
\hline Never & 9.7 & 17.4 & 0.0 & 2.1 & 90.6 & 20.7 \\
\hline 1 time per month or rarely & 22.6 & 29.8 & 14.0 & 5.7 & 9.4 & 16.4 \\
\hline 2-3 times per month & 34.1 & 30.4 & 50.7 & 17.8 & 0.0 & 32.6 \\
\hline Several times per week & 33.6 & 22.5 & 35.4 & 74.3 & 0.0 & 30.3 \\
\hline
\end{tabular}

according to the level of agreement declared by respondents with the statement "I like to feel and live my body very intensely." Again, we aggregated responses differentiating among disagree (completely or rather), indifference or agree (completely or rather). The categorisations used for our key explanatory variables allowed us to reach the double advantage of preserving the sample size of each category and juxtaposing meaningful levels of (dis)agreement or (dis)satisfaction. We ran the regression model also considering the response variables in their original disaggregation, but the results proved to be consistent. These results are not presented here but are available upon request.

The association between psychological factors and sexual risk profiles was estimated net of a set of socio-demographic characteristics of Italian youths. These characteristics were as follows: age (18-20; 21-22; 23-26), living arrangement (dichotomized between with at least one parent and alone, with friends or partner), area of residence (North; Centre; South/Islands) and, to account for family background, the mother's educational level (low; medium; high) and parental separation (yes; no). In our sample, less than $3 \%$ defined their sexual orientation as homosexual or bisexual; this proportion is virtually the same as in the complete sample. Unfortunately, the percentage of homosexual and bisexual participants was too low to attempt any further exploration of this issue.

Gender differences in the relationship between psychological characteristics (body satisfaction, self-esteem and sensation seeking) and sexual risk profiles were investigated through interactions. The likelihood ratio chi-squared test proved a significant fit for the model (LR $\left.x^{2}=890.16, p<.000\right)$, indicating that our full model predicted 
significantly well (e.g., Agresti, 2002) and thus proving the effectiveness of an approach that accounts for gender differences.

A description of our sample in terms of the aforementioned psychological and sociodemographic characteristics is offered in Table 2 in the Appendix. The final model refers to 5207 individuals $(51.5 \% \mathrm{men})$. The model has been estimated considering all these individuals, notwithstanding a reduced percentage of missing values on some characteristics that are assumed to be randomly distributed. Results are presented in terms of predicted probabilities to be classified in a given group depending on one's body satisfaction, selfesteem and sensation seeking. Model coefficients are reported in Table 2 in the Appendix.

\section{Results}

\section{Sexual risk profiles of Italian students}

The cluster analysis performed led us to classify individuals into five groups according to the riskiness of their sexual behaviours. Table 1 shows the distribution of the responses with respect to the sexual behaviours that were considered to create the clusters.

The first two clusters included individuals characterised by highly hazardous sexual behaviours, both recent and in the more distant past. Specifically, the first cluster (\#1, about $18 \%$ of our sample) grouped individuals who had multiple sexual partners in a proportion higher than the average (80\% of them had 6 or more partners, against an average value of $16 \%)$ and had their first intercourse at younger ages (16 years old or before). In this group, a higher percentage than the average did not use any contraceptive method either in their first or last sexual intercourse. Among the possible contraceptive methods, a condom (i.e., the unique method able to prevent infective diseases) was not or was rarely used. Twentyfive percent of people classified in this cluster declared they had frequently had sexual intercourse within uncommitted relationships without using condoms, relative to the $10 \%$ average in the total sample. People grouped in this cluster had frequent sexual intercourses with a person who was not their partner, without feeling affection for the partner or had paid for sex. We labelled this cluster as the "promiscuous, high-risk" cluster.

The second cluster (\#2) was similar to the previous one in terms of contraceptive use but was characterised by a lower number of sexual partners, betrayal or recourse to paid sex; hence, a lower level of promiscuity was present among these individuals. We called this group, which constituted $15 \%$ of the sample, the "risk-taking" cluster.

The third cluster (\#3) was the largest, constituting about $40 \%$ of our sample. This cluster, labelled as "few partners, low risk," included those who had 1-2 sexual partners in their lives and started sexual relationships with some delay, at age 17 or later. Most of these respondents used condoms both in their first and most recent intercourse, talked with their partners about contraception prior to or during their first sexual experiences and declared to have always used condoms during intercourse with casual partners; they declared they had rarely paid for sex, betrayed a partner or had sex without love. These respondents had regular sexual activity in the last 3 months before the interview, probably with a stable partner.

The most important discriminant feature of the fourth cluster (\#4, which represented $9 \%$ of the sample) was that only $22 \%$ of respondents in this cluster declared they had used a condom in the most recent intercourse, whereas more than $50 \%$ opted for a pill or a patch. A deeper investigation led us to the conclusion that this group included those 
individuals who were in stable relationships during the survey and felt engaged with their current partners. Nevertheless, these respondents took some risks in their sexual experiences, as they reported they did not always use condoms with casual partners. The label "monogamous, medium risk" conveys the main characteristics of this cluster.

Finally, we found an interesting fifth cluster (\#5) accounting for about $18 \%$ of the sample. Respondents classified in this cluster had a "late" start of sexual intercourse and generally a low number of partners; they generally tended to adopt safe behaviours, as did those in the third cluster. The main aspect characterising this group was that these respondents did not have intense sexual lives, at least in terms of frequency. They declared they did not have sexual intercourse during the last 3 months before the survey. We labelled them the "low sexual practice" cluster.

\section{Psychological correlates of sexual risk profiles}

The core of our analysis was the estimation of associations between the identified risktaking profiles and the individual psychological characteristics we investigated, accounting for potential gender differences. For this reason, our model specification included interactions between gender and these three variables. The value of these interactions was accounted for in the predicted probabilities (PPs), while the other control variables (namely, age, living arrangement, area of residence, mother's educational level, parental separation) were set to their reference level (less than 21 years old, living with at least one parent, Northern Italy, low, no). All the estimated coefficients are reported in Table 3 of the Appendix. For the sake of brevity, in the following, we highlight only the most interesting associations, neglecting not significant ones.

In consideration of the fundamental features of each cluster, it seems that women were overall significantly less likely to be classified in the two high sexual risk clusters (PPs equal to 0.11 for both clusters \#1 and \#2, compared to 0.24 and 0.19 as for men; see Figure 2 in the Appendix), regardless of their scores on the three psychological variables. Instead, young Italian women had the highest probability (0.50), significantly higher than that of men (0.30), to be classified in cluster \#3. A slightly higher probability for women was found also for cluster \#4, whereas no gender differences were depicted with regard to cluster \#5. It thus seems that women on average tend to be safer and more prudent than men are in their sexual lives.

Given this overall gender difference, we will now examine each cluster's characteristics, considering the likelihood of being classified in each cluster depending on the answers given to the three psychological factors considered (Fig. 1). We clearly observe that the more men were satisfied with their bodies, the higher their probability was to be classified into cluster \#1 (the black line in graph a, that is the confidence intervals for not satisfied boys, $\mathrm{PP}=0.21$, and satisfied ones, $\mathrm{PP}=0.27$, do not overlap). Moreover, those men who declared wanting to feel and live in their body intensely (our proxy for body sensation seeking) were more likely to have a non-safe sexual life (i.e., their probability to be classified in cluster \#1 rises to 0.25 ; the black line in graph c). On the contrary, for women, body satisfaction is not significantly related to the probability of being classified in cluster \#1. However, we depicted a modest negative gradient (significant only at the $10 \%$ level) between women's body satisfaction and the probability of being in cluster \#2 (the grey line in graph d; PP decreases from 0.13 to 0.09 as the level of satisfaction increases). 


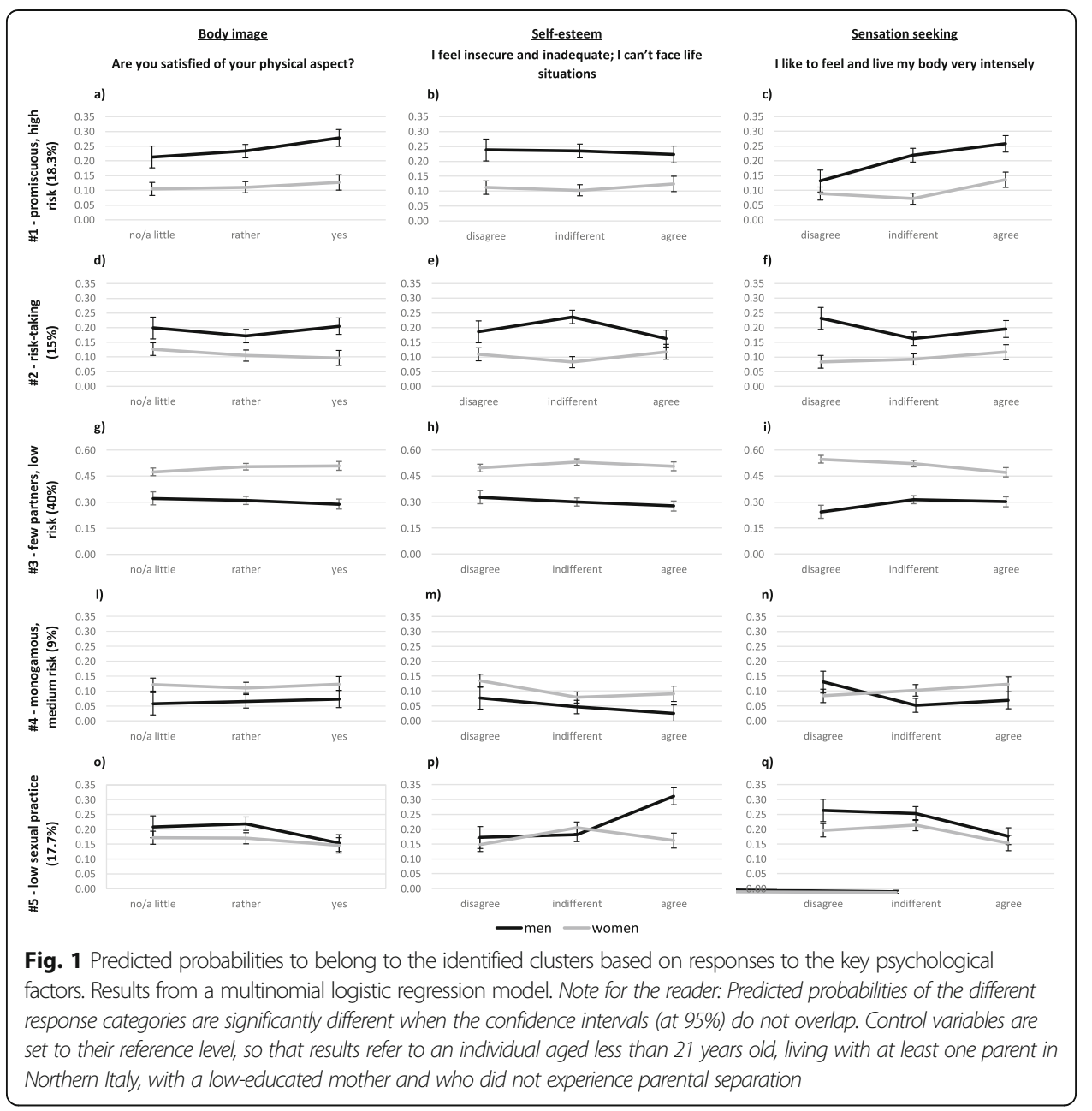

In cluster \#3, apart from the gendered likelihood of classification highlighted before, we detected a distinct pattern for men and women. High body satisfaction increased the probability for women to be classified in this cluster (from 0.47 to 0.50 ; the grey line in graph g), whereas high levels of sensation seeking diminished this probability (from 0.55 to 0.47 ; the grey line in graph i). On the contrary, men who had higher levels of selfesteem and liked to feel and live in their bodies intensely had a higher probability of being classified into this cluster (the black lines in graphs $\mathrm{h}$ and $\mathrm{i}$, which correspond, respectively, to PPs equal to 0.33 and 0.30). Body satisfaction was not relevant for men (graph g). The other cluster characterised by low-medium risk was cluster \#4, which was less numerous and had no detectable specific patterns, either in terms of gender or psychological differences (graphs $1-n)$. It is worth noting that self-esteem seems to be the least relevant aspect in classifying people into these four clusters, either for men or women.

Finally, cluster \#5, characterised by young people who seemed to have scarce and infrequent sexual activity, presented interesting insights (graphs o-q), especially along gender lines. Men who were not at all satisfied or had low satisfaction with their bodies, who felt insecure and inadequate and who did not love feeling and living their bodies intensely had a significantly higher probability of being classified into this cluster in comparison to their satisfied, highly confident and sensation-seeking counterparts (the black lines; PPs 
respectively equal to $0.21,0.36$ and 0.26 ). As for young women (the grey lines), the correlation is less clear: women who liked living their body intensely had a lower probability to be classified in this cluster ( $\mathrm{PP}=0.20$ ), but self-esteem and body image are, surprisingly, not discriminant for them. The investigated psychological characteristics seem thus to be especially relevant to the sexual functioning of boys but not of girls.

The estimates of the socio-demographic correlates of sexual risk profiles are interesting too (Table 2 in the Appendix). The likelihood to be classified in the two highly risky clusters (\#1 and \#2) increases as age increases, as well as for cluster \#4, that identifying people in stable couples. It is interesting to see that people living alone, with friends, or with partners have a higher probability than those living with parents to be classified both in the higher-risk clusters (\#1) and in the low sexual practice one (\#5). Further investigation could deepen this link; however, the limited sample size prevents this differentiation. Apart from a somewhat lower likelihood for young people living in Southern Italy to be classified in clusters \#4 and \#5, no strong territorial differences are detected. This probably highlights a tendency toward a uniformity of sexual behaviours across Italy. Finally, respondents with more highly educated mothers seem to be more promiscuous and more prone to adopting risky behaviours in their sexual lives.

\section{Data limitations}

Some clarifications about the data used are required before conclusions. First, SELFY is extraordinarily rich in terms of information to investigate opinions, attitudes and behaviours of young Italians (for instance, on sentimental relationships, sexual life, religiosity, free time and risky behaviours). This questionnaire provides a large sample size not often available for specific and detailed surveys. The sampling approach adopted (Dalla Zuanna et al,, 2019), however, did not enable us to generalise our findings to all Italian youth; the available information only described undergraduate students in economics and statistics in Italian public universities. Indeed, other studies in the Italian context showed that less educated youth or those who chose other educational fields tended to be more sexually precocious than the average of our sample (ibid.). We thus argue that, if a selection bias exists due to the specific group under investigation, it might operate in a conservative way, thus necessitating the monitoring and scrutinising of risk-taking behaviours among young Italians.

Second, we are aware that a potential limitation for our study could stem from the fact that the self-reported personality characteristics we considered here are measured through single-item variables and not through fully validated instruments. Nevertheless, some research argued that single-item measures can be useful when a holistic impression is informative: if the focus of the researcher is on the individual as a whole and on his/her perception of a specific concept, a single-item indicator can be considered an appropriate measurement method (Youngblut \& Casper, 1993). Our aim was neither to measure specific levels of the psychological characteristics considered nor to assess the importance of different dimensions in these concepts; rather, our objective was to determine if and to what extent large variations in these perceptions could be associated with sexual (risky) behaviours. Overall, our study highlighted the need to collect comprehensive and multidimensional data not only on sexual behaviours of Italian youth but also on psychological characteristics that could be related to these behaviours. Moreover, future studies should apply more valid and reliable multi-item scales to assess these constructs, also applying 
different methodologies, such as vignettes and scenarios (i.e., a technique of data collection which, by presenting hypothetical situations, could be particularly useful when dealing with sensitive topics, such as sexual behaviour; see, for example Hughes, 1998; Lapatin et al., 2012), to reduce possible bias due to self-assessed measures.

Finally, we must mention that we selected a sub-sample of people who already had sexual intercourse at least once in their lives because our aim was to investigate the psychological correlates of sexual risk behaviours. In that sense, individuals who never had sexual intercourse, by definition, do not engage in risky behaviours. We are conscious that emerging adults who are not sexually active could be characterised by different views of their own bodies or other emotional aspects, and a deeper investigation of this group deserves more attention.

\section{Discussion}

Unsafe sexual behaviours represent the major determinants for STI transmission. Recent data about infection diffusions (e.g., European Centre for Disease Prevention and Control, 2017; Italian National Institute of Health, 2018a, 2018b) call for more attention to the factors that can predict sexual risk taking. In this paper, we investigated if and how some individuals' psychological characteristics, namely, body satisfaction, selfesteem and body sensation seeking, were associated with unsafe and dangerous sexual behaviours among Italian young adults. To the best of our knowledge, these factors have been scarcely examined within the Italian context. We utilised SELFY, a large survey concerning the opinions, attitudes and behaviours of young Italians.

Several key points may be highlighted from our study. First, we found that risky sexual behaviours are still widespread among Italian young people. The two clusters we identified as high sexual risk taking (\#1 and \#2) constituted one third of our sample, a non-negligible proportion. Differences between the two clusters were present, but both were characterised, to a greater extent than the average, by a reduced use of condoms, especially with casual partners; sexual intercourse with unknown persons; and infrequent dialogue with their partners about contraception. Even if it is true that these results cannot be generalised to the Italian young population as a whole, our findings provided evidence that further public efforts in continuing sexual education are needed to promote responsible sexual practices.

Second, we proved that women are generally less inclined to adopt risk-taking behaviours in sexual life, consistent with previous research (Vasilenko et al., 2015). A recent study by Dalla Zuanna et al. (2019), using the same data we did, considered opinions and sexual behaviours separately, arguing that a convergence pattern between young men and women is in act in Italy. We agree that a sort of "masculinisation of girls' sexual life courses in terms of their opinions and behaviours outside a couple relationship" (ibid., p. 16) plays a role, especially in some aspects of sexual life, such as the number of sexual partners or having casual partners. Nevertheless, when opting for a comprehensive account of several risky sexual behaviours simultaneously, thus highlighting global profiles of sexual risk exposure, it seems that convergence toward a risk-taking behavioural model is still far from reachable, although young Italian women have started to relax some social norms regarding sexual life.

The core of our study was to investigate if and to what extent some individual psychological characteristics-namely body satisfaction, self-esteem and sensation seeking-were 
associated with sexual risk profiles. Bearing in mind the (still) persisting male and female differences in various sex domains, we explored potential gendered patterns in this association. Our findings showed that these variables are differently linked to sexual risk taking according to gender. In line with our predictions, our analyses showed that a positive view of one's body (i.e., body satisfaction) was associated with a higher probability of adopting risky (and promiscuous) sexual behaviours among young men. These findings are in line with previous international data (Gillen et al., 2006) that suggest that men who like their bodies are likely to feel more confident with their muscularity, which is stereotypically linked to sexual freedom and power (Matera et al., 2019). In addition, as we predicted, men who like themselves might be more resolute in satisfying their own needs, such as the achievement of pleasure, which is allegedly heightened when a condom is not used (Randolph et al., 2007). In contrast, a significant link between body satisfaction and sexual risk taking did not emerge for Italian women, even though weak evidence concerning a negative association between these variables was found.

Consistent with previous findings (Gullette and Lyons, 2005; McCoul and Haslam, 2001; Spitalnick et al., 2007; Voisin et al., 2013), sensation seeking showed a certain relevance in defining sexual risk profiles. Young Italians, especially men, who liked to feel intense emotions related to their bodies intensely (the so-called sensation-seeking individuals) tended to have highly frequent sexual intercourse, also with unknown partners, a higher number of sexual partners and less frequent condom use, proving the importance of considering personality characteristics in the study of sexual risk exposure (Kalichman et al., 1994).

Finally, our analyses led to the identification of one last cluster of individuals (\#5). These young people did not reveal high sexual risk taking but rather seemed to be characterised by a sporadic sexual life. Previous literature found that those who do not feel at ease with their bodies tend to associate sexual activity with negative emotions (e.g., embarrassment and shame), thus compromising their sexual lives (La Rocque and Cioe, 2011). Our findings on the three psychological dimensions explored support this view, especially for young men. Even if no sexual risk taking was detected in this group, this pattern raises concerns about the possible negative consequences of sexual functioning and satisfaction among individuals with low body satisfaction and low self-esteem.

We think that this study represents an initial but pivotal attempt to bring to light the need for public discussion of psychosocial and behavioural variables that can predict sexual risk taking among Italian youths. In this regard, our research has relevant implications for prevention/intervention programs about sexual health targeting young adults in Italy. Effective STI prevention campaigns and interventions should be targeted and tailored based on the characteristics of those who are involved in risky sexual behaviours. Although our study does not examine causation, it nevertheless suggests that body image and self-worth could be relevant areas of intervention. Notably, communication and education strategies on these issues should be different for women and men. For young women, strategies might focus on promoting satisfaction with one's body and self-esteem while removing barriers (whether mental or physical) that tend to interfere with safe sexual behaviours. Women should be helped to recognise their value, which might positively affect their sexual health. For men, social campaigns should convey the message that self-worth should correspond to self-protection rather than to hazardous behaviours. While it is important to value oneself, it could be dangerous to 
feel invincible. As Liuccio et al. (2019) suggested, the university environment could be especially suitable for offering cues to action (Rosenstock et al., 1994) and shaping social norms that can foster healthy sexual behaviours.

\section{Appendix}

Table 2 Distribution of the socio-demographic and psychological characteristics included in the multinomial logistic regression model

\begin{tabular}{|c|c|c|c|c|}
\hline & \multirow{3}{*}{$\begin{array}{l}\text { Men } \\
N=2682 \\
\% \text { val. }\end{array}$} & \multirow{3}{*}{$\begin{array}{l}\text { Women } \\
N=2525 \\
\% \text { val. }\end{array}$} & \multirow{2}{*}{\multicolumn{2}{|c|}{$\frac{\text { Total }}{N=5207}$}} \\
\hline & & & & \\
\hline & & & $\%$ val. & abs. val. \\
\hline \multicolumn{5}{|l|}{ Classes of age } \\
\hline $18 / 20$ & 33.7 & 38.2 & 35.9 & 1870 \\
\hline $21 / 22$ & 48.4 & 47.2 & 47.8 & 2489 \\
\hline $23 / 26$ & 17.9 & 14.6 & 16.3 & 848 \\
\hline \multicolumn{5}{|l|}{ Living arrangement } \\
\hline With at least one parent & 67.4 & 63.7 & 65.6 & 3416 \\
\hline Alone, with friends or partner & 24.3 & 27.2 & 25.7 & 1339 \\
\hline n.a. & 8.3 & 9.1 & 8.7 & 452 \\
\hline \multicolumn{5}{|l|}{ Area of residence } \\
\hline North & 34.4 & 31.4 & 33.0 & 1716 \\
\hline Centre & 27.3 & 28.4 & 27.8 & 1449 \\
\hline South/islands & 38.4 & 40.1 & 39.2 & 2042 \\
\hline \multicolumn{5}{|l|}{ Mother's educational level } \\
\hline Low & 18.2 & 27.0 & 22.5 & 1172 \\
\hline Medium & 55.9 & 52.8 & 54.4 & 2834 \\
\hline High & 24.7 & 19.1 & 22.0 & 1145 \\
\hline n.a. & 1.1 & 1.0 & 1.1 & 56 \\
\hline \multicolumn{5}{|l|}{ Parental separation } \\
\hline No & 84.5 & 85.4 & 84.9 & 4421 \\
\hline Yes & 13.9 & 13.4 & 13.7 & 713 \\
\hline n.a. & 1.6 & 1.2 & 1.4 & 73 \\
\hline \multicolumn{5}{|c|}{ Satisfaction with a physical aspect } \\
\hline At all or little satisfied & 17.2 & 32.9 & 24.8 & 1291 \\
\hline Rather satisfied & 46.8 & 41.9 & 44.4 & 2314 \\
\hline Satisfied & 35.2 & 24.6 & 30.1 & 1565 \\
\hline n.a. & 0.8 & 0.6 & 0.7 & 37 \\
\hline \multicolumn{5}{|l|}{ Feeling of insecurity } \\
\hline Indifferent & 13.7 & 12.9 & 13.3 & 692 \\
\hline Disagree & 68.5 & 65.8 & 67.2 & 3498 \\
\hline Agree & 12.1 & 17.3 & 14.6 & 760 \\
\hline n.a. & 5.8 & 4.0 & 4.9 & 257 \\
\hline \multicolumn{5}{|l|}{ Sensation seeking } \\
\hline Indifferent & 21.2 & 21.8 & 21.5 & 1120 \\
\hline Disagree & 2.6 & 6.9 & 4.7 & 245 \\
\hline Agree & 70.0 & 65.7 & 67.9 & 3535 \\
\hline n.a. & 6.2 & 5.6 & 5.9 & 307 \\
\hline
\end{tabular}


Table 3 Coefficients estimated through a multinomial logistic regression model for the probability to belong to the 5 clusters of sexual risk profile. Cluster \#3 (few partners, low risk) is the outcome reference category

\begin{tabular}{|c|c|c|c|c|c|c|c|c|}
\hline & \multicolumn{2}{|c|}{$\begin{array}{l}\text { Outcome = \#1 } \\
\text { promiscuous, } \\
\text { high risk }\end{array}$} & \multicolumn{2}{|c|}{$\begin{array}{l}\text { Outcome = \#2 } \\
\text { risk taking }\end{array}$} & \multicolumn{2}{|c|}{$\begin{array}{l}\text { Outcome = \#4 } \\
\text { monogamous, } \\
\text { medium risk }\end{array}$} & \multicolumn{2}{|c|}{$\begin{array}{l}\text { Outcome }=\# 5 \\
\text { low sexual practice }\end{array}$} \\
\hline & Coef. & $p$-value & Coef. & $p$-value & Coef. & $p$-value & Coef. & $p$-value \\
\hline \multicolumn{9}{|l|}{ Gender (ref.: male) } \\
\hline Female & -1.731 & 0.000 & -1.453 & 0.000 & 0.284 & 0.530 & -0.385 & 0.160 \\
\hline \multicolumn{9}{|l|}{ Classes of age (ref.: 18-20) } \\
\hline $21-22$ & 0.348 & 0.000 & 0.285 & 0.003 & 0.048 & 0.676 & -0.046 & 0.607 \\
\hline $23-26$ & 1.320 & 0.000 & 0.620 & 0.000 & 0.605 & 0.000 & 0.177 & 0.177 \\
\hline \multicolumn{9}{|c|}{ Living arrangement (ref.: with at least one parent) } \\
\hline Alone, with friends or partner & 0.536 & 0.000 & 0.262 & 0.010 & 0.245 & 0.043 & 0.282 & 0.003 \\
\hline \multicolumn{9}{|l|}{ Area of residence (ref.: north) } \\
\hline Centre & 0.051 & 0.641 & 0.099 & 0.385 & -0.113 & 0.396 & -0.123 & 0.240 \\
\hline South/islands & 0.011 & 0.914 & 0.033 & 0.758 & -0.272 & 0.031 & -0.339 & 0.001 \\
\hline \multicolumn{9}{|c|}{ Mother's educational level (ref.: low) } \\
\hline Medium & 0.108 & 0.313 & 0.204 & 0.068 & 0.212 & 0.104 & 0.075 & 0.459 \\
\hline High & 0.474 & 0.000 & 0.273 & 0.041 & 0.142 & 0.381 & 0.127 & 0.302 \\
\hline \multicolumn{9}{|l|}{ Parental separation (ref.: no) } \\
\hline Yes & 0.513 & 0.000 & 0.426 & 0.001 & 0.342 & 0.020 & -0.033 & 0.799 \\
\hline \multicolumn{9}{|c|}{ Satisfaction with physical aspect (ref.: at all or little satisfied) } \\
\hline Rather satisfied & 0.133 & 0.400 & -0.110 & 0.500 & 0.178 & 0.496 & 0.087 & 0.580 \\
\hline Satisfied & 0.399 & 0.016 & 0.150 & 0.381 & 0.374 & 0.165 & -0.200 & 0.259 \\
\hline \multicolumn{9}{|c|}{ Feeling of insecurity (ref.: indifferent) } \\
\hline Disagree & -0.074 & 0.648 & -0.328 & 0.046 & 0.413 & 0.142 & -0.139 & 0.419 \\
\hline Agree & 0.011 & 0.962 & -0.306 & 0.184 & -0.550 & 0.228 & 0.632 & 0.003 \\
\hline \multicolumn{9}{|l|}{ Sensation seeking } \\
\hline Disagree & -0.264 & 0.546 & 0.607 & 0.102 & 1.174 & 0.014 & 0.289 & 0.419 \\
\hline Agree & 0.219 & 0.113 & 0.229 & 0.119 & 0.334 & 0.142 & -0.334 & 0.013 \\
\hline \multicolumn{9}{|c|}{ Satisfaction with physical aspect by gender (ref.: male not satisfied) } \\
\hline Female rather satisfied & -0.151 & 0.514 & -0.143 & 0.531 & -0.335 & 0.275 & -0.157 & 0.447 \\
\hline Female satisfied & -0.277 & 0.267 & -0.495 & 0.053 & -0.432 & 0.182 & -0.034 & 0.887 \\
\hline \multicolumn{9}{|c|}{ Feeling of insecurity by gender (ref.: male indifferent) } \\
\hline Female disagreeing & 0.245 & 0.376 & 0.679 & 0.015 & 0.205 & 0.575 & -0.128 & 0.589 \\
\hline Female agreeing & 0.247 & 0.473 & 0.714 & 0.040 & 0.752 & 0.161 & -0.823 & 0.004 \\
\hline \multicolumn{9}{|c|}{ Sensation seeking by gender (ref.: male indifferent) } \\
\hline Female disagreeing & 0.441 & 0.424 & -0.749 & 0.123 & -1.425 & 0.014 & -0.422 & 0.322 \\
\hline Female agreeing & 0.551 & 0.023 & 0.124 & 0.585 & -0.041 & 0.885 & 0.100 & 0.597 \\
\hline Constant & -1.374 & 0.000 & -0.955 & 0.000 & -2.558 & 0.000 & -0.211 & 0.374 \\
\hline
\end{tabular}




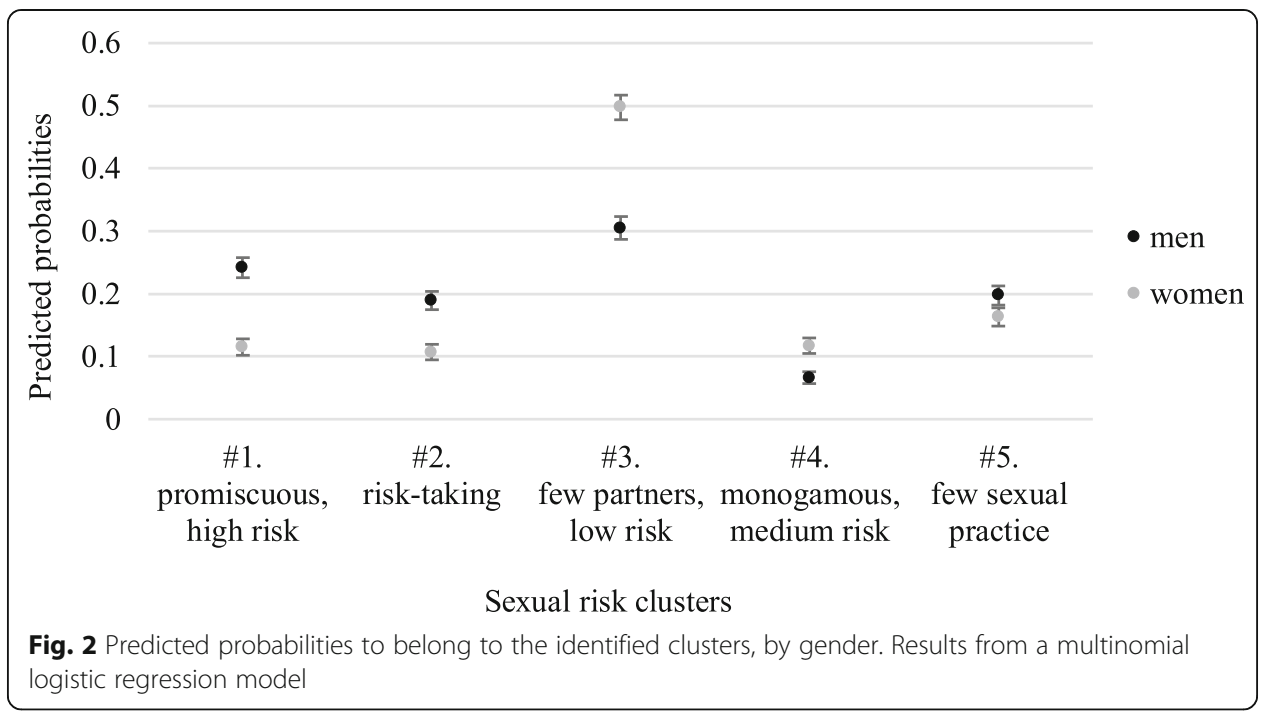

\section{Abbreviations}

STI: Sexually transmitted infections; ISS: Italian National Institute of Health; ECDC: European Centre for Disease Prevention and Control; SELFY: Sexual and Emotional LiFe of Youths

\section{Acknowledgements}

None

\section{Authors' contributions}

The paper is the result of the collaboration between authors, and each author, EP and CM, equally contributed to all parts of the manuscript. The authors read and approved the final manuscript.

\section{Funding}

No external funding has been benefitted for this study.

\section{Availability of data and materials}

The authors declare that the dataset analysed in the current study are available from the corresponding author on reasonable request.

\section{Competing interests}

The authors declare that they have no competing interests.

\section{Author details}

${ }^{1}$ Dipartimento di Statistica, Informatica, Applicazioni - DiSIA, University of Florence, Viale Morgagni 59, 50134 Firenze, Italy. ${ }^{2}$ Dipartimento di Formazione, Lingue, Intercultura, Letterature e Psicologia - FORLILPSI, University of Florence, Via di San Salvi, 12 - Pad. 26, 50135 Firenze, Italy.

Received: 10 December 2019 Accepted: 16 May 2020

Published online: 10 June 2020

\section{References}

Agresti, A. (2002). Categorical data analysis. Hoboken: Wiley.

Arnett, J. J. (2000). Emerging adulthood: A theory of development from the late teens through the twenties. American psychologist, 55(5), 469-480.

Arnold, P., Fletcher, S., \& Farrow, R. (2002). Condom use and psychological sensation seeking by college students. Sexual and Relationship Therapy, 17(4), 355-365.

Beadnell, B., Morrison, D. M., Wilsdon, A., Wells, E. A., Murowchick, E., Hoppe, M., Rogers Gillmore, M., \& Nahom, D. (2005). Condom use, frequency of sex, and number of partners: Multidimensional characterization of adolescent sexual risktaking. The Journal of Sex Research, 42(3), 192-202.

Biemmi, I. (2015). Gender in schools and culture: Taking stock of education in Italy. Gender and Education, 27(7), 812-827. Blashill, A., \& Safren, S. (2015). Body dissatisfaction and condom use self-efficacy: A meta-analysis. Body Image, 12, 73-77. Boden, J. M., \& Horwood, L. J. (2006). Self-esteem, risky sexual behavior, and pregnancy in a New Zealand birth cohort. Archives of Sexual Behavior, 35(5), 549-560

Brady, J. P., Nogg, K. A., Rozzell, K. N., Rodriguez-Diaz, C. E., Horvath, K. J., Safren, S. A., \& Blashill, A. J. (2019). Body image and condomless anal sex among Young Latino sexual minority men. Behaviour research and therapy, 115, 129-134.

Brooks-Gunn, J., \& Furstenberg, F. (1989). Adolescent sexual behavior. American Psychologist, 44, 249-257.

Cash, T. F., \& Pruzinsky, T. (2002). Body image: A handbook of theory, research, and clinical practice. New York: Guilford Press. 
Chawla, N., \& Sarkar, S. (2019). Defining "high-risk sexual behavior" in the context of substance use. Journal of Psychosexual Health, 1(1), 26-31.

Dalla Zuanna, G., Caltabiano, M., Minello, A., \& Vignoli, D. (2019). Catching up! The sexual opinions and behaviour of Italian students (2000-2017). DiSIA Working Paper 2019/02.

De Looze, M., Madkour, A. S., Huijts, T., Moreau, N., \& Currie, C. (2019). Country-level gender equality and adolescents' contraceptive use in Europe, Canada and Israel: Findings from 33 countries. Perspectives on sexual and reproductive health, 51(1), 43-53.

Durbin, M., DiClemente, R. J., Siegel, D., Krasnovsky, F., Lazarus, N., \& Camacho, T. (1993). Factors associated with multiple sex partners among junior high school students. Journal of Adolescent Health, 14, 202-207.

Eisenberg, M. E., Neumark-Sztainer, D., \& Lust, K. D. (2005). Weight-related issues and high-risk sexual behaviors among college students. Journal of American College Health, 54(2), 95-101.

Enejoh, V., Pharr, J., Mavegam, B. O., Olutola, A., Karick, H., \& Ezeanolue, E. E. (2016). Impact of self-esteem on risky sexual behaviors among Nigerian adolescents. AIDS Care, 28(5), 672-676.

Ethier, K. A., Kershaw, T. S., Lewis, J. B., Milan, S., Niccolai, L. M., \& Ickovics, J. R. (2006). Self-esteem, emotional distress and sexual behavior among adolescent females: Inter-relationships and temporal effects. Journal of Adolescent health, 38(3), $268-274$.

European Centre for Disease Prevention and Control (2017). HIV/AIDS surveillance in Europe 2017-2016 data, ECDC: Stockholm, Sweden. Retrieved from https:/ecdc.europa.eu/en/publications-data/hivaids-surveillance-europe-2017-2016-data.

Everitt, B. S. (1974). Cluster Analysis, Heinemann.

Gholizadeh, S., Rooney, B. M., Merz, E. L., Malcarne, V. L., Safren, S. A., \& Blashill, A. J. (2018). Body image and condomless anal sex among sexual minority men living with HIV. AIDS and behavior, 22(2), 658-662.

Gillen, M., Lefkowitz, E., \& Shearer, C. (2006). Does body image play a role in risky sexual behavior and attitudes? Journal of Youth and Adolescence, 35, 243-255.

Goodson, P., Buhi, E. R., \& Dunsmore, S. C. (2006). Self-esteem and adolescent sexual behaviors, attitudes, and intentions: A systematic review. Journal of Adolescent Health, 38(3), 310-319.

Gullette, D. L., \& Lyons, M. A. (2005). Sexual sensation seeking, compulsivity, and HIV risk behaviors in college students. Journal of community health Nursing, 22(1), 47-60.

Hughes, R. (1998). Considering the vignette technique and its application to a study of drug injecting and HIV risk and safer behaviour. Sociology of Health \& IIIness, 20,381-400.

Italian National Institute of Health (2018a). Notiziario dell'Istituto Superiore di Sanità, Vol. 31, N. 6, June 2018, ISSN 1827-6296 (online).

Italian National Institute of Health (2018b). Notiziario dell'Istituto Superiore di Sanità, Vol. 31, N. 9, Supplement 1-2018, ISSN 1827-6296 (online).

Jain, A. K. \& Dubes, R. C. (1988). Algorithms for clustering data. Prentice-Hall.

Kalichman, S. C., Johnson, J. R., Adair, V., Rompa, D., Multhauf, K., \& Kelly, J. A. (1994). Sexual sensation seeking: Scale development and predicting AIDS-risk behavior among homosexually active men. Journal of personality assessment, 62(3), 385-397.

Kaufman, L. \& Rousseeuw, P. J. (2005). Finding groups in data: an introduction to cluster analysis. Hoboken: Wiley.

Kim, D. H., Bassett, S. M., Takahashi, L., \& Voisin, D. R. (2018). What does self-esteem have to do with behavioral health among low-income youth in Chicago? Journal of Youth Studies, 21(8), 999-1010.

Kiviruusu, O., Konttinen, H., Huurre, T., Aro, H., Marttunen, M., \& Haukkala, A. (2016). Self-esteem and body mass index from adolescence to mid-adulthood. A 26-year follow-up. International journal of behavioral medicine, 23(3), 355-363.

La Rocque, C. L., \& Cioe, J. (2011). An evaluation of the relationship between body image and sexual avoidance. Journal of sex research, 48(4), 397-408

Lapatin, S., Gonçalves, M., Nillni, A., Chavez, L., Quinn, R. L., Green, A., \& Alegría, M. (2012). Lessons from the use of vignettes in the study of mental health service disparities. Health Services Research, 47, 1345-1362.

Liuccio, M., Borgia, C., Chiappetta, M., Martino, B., \& Giordano, F. (2019). The condom use among young adults and its determinants: An Italian study. La Clinica Terapeutica, 170(4), e278-e284.

Luster, T., \& Small, S. A. (1994). Factors associated with sexual risk-taking behaviors among adolescents. Journal of Marriage and Family, 56(3), 622-632.

Matera, C. (2014). Encouraging safer sex: Mediating and moderating effects among Italian girls. International Journal of Sexual Health, 26, 217-228.

Matera, C., Nerini, A., Baroni, D., \& Stefanile, C. (2018). What messages can foster safer sex among young women? Experimental evidence concerning the role of emotions and moral norms. Psychology, health \& medicine, 23(6), 741-751.

Matera, C., Nerini, A., \& Stefanile, C. (2019). Sexual orientation, peer influence, body dissatisfaction, and Eudaimonic well-being in Italian men. Frontiers in psychology, 10, 1843.

McAuley, E., Blissmer, B., Katula, J., Duncan, T. E., \& Mihalko, S. L. (2000). Physical activity, self-esteem, and self-efficacy relationships in older adults: A randomized controlled trial. Annals of Behavioral Medicine, 22(2), 131.

McCoul, M. D., \& Haslam, N. (2001). Predicting high risk sexual behaviour in heterosexual and homosexual men: The roles of impulsivity and sensation seeking. Personality and individual differences, 31(8), 1303-1310.

McGee, R. O. B., \& Williams, S. (2000). Does low self-esteem predict health compromising behaviours among adolescents? Journal of adolescence, 23(5), 569-582.

McNair, L. D., Carter, J. A., \& Williams, M. K. (1998). Self-esteem, gender, and alcohol use: Relationships with HIV risk perception and behaviors in college students. Journal of sex \& marital therapy, 24(1), 29-36.

Merianos, A. L., King, K. A., \& Vidourek, R. A. (2013). Body image satisfaction and involvement in risky sexual behaviors among university students. Sexuality \& Culture, 17(4), 617-630.

Nerini, A., \& Matera, C. (2020). Oggettivazione corporea, norme morali e rapporti sessuali a rischio: canoni morali e canoni estetici in uomini e donne eterosessuali. La Camera blu, 21, 77-97.

Norton, T. R., Bogart, L. M., Cecil, H., \& Pinkerton, S. D. (2005). Primacy of affect over cognition in determining adult men's condom-use behavior: A review 1. Journal of Applied Social Psychology, 35(12), 2493-2534.

Olaoye, T., \& Agbede, C. (2019). Prevalence and personal predictors of risky sexual behaviour among in-school adolescents in the Ikenne Local Government Area, Ogun State, Nigeria. International journal of adolescent medicine and health, online first, November 12, 2019. doi: https://doi.org/https://doi.org/10.1515/ijamh-2019-0135 
Potard, C., Lancelot, C., \& Courtois, R. (2019). Examining relationships between sexual risk-safety behaviors and physical selfconcept by gender: A cluster analytical approach. Emerging Adulthood, 7(1), 31-44.

Preston, D. B., D'augelli, A. R., Kassab, C. D., Cain, R. E., Schulze, F. W., \& Starks, M. T. (2004). The influence of stigma on the sexual risk behavior of rural men who have sex with men. AIDS Education \& Prevention, 16(4), 291-303.

Randolph, M. E., Pinkerton, S. D., Bogart, L. M., Cecil, H., \& Abramson, P. R. (2007). Sexual pleasure and condom use. Archives of sexual behavior, 36(6), 844-848.

Ritchwood, T. D., Ford, H., DeCoster, J., Sutton, M., \& Lochman, J. E. (2015). Risky sexual behavior and substance use among adolescents: A meta-analysis. Children and youth services review, 52, 74-88.

Rolison, M. R., \& Scherman, A. (2002). Factors influencing adolescents' decisions to engage in risk-taking behavior. Adolescence, 37(147), 585.

Rosenberg, M., Schooler, C., Schoenbach, C., \& Rosenberg, F. (1995). Global self-esteem and specific self-esteem: Different concepts, different outcomes. American Sociological Review, 60(1), 141-156.

Rosenstock, I. M., Strecher, V. J., \& Becker, M. H. (1994). The health belief model and HIV risk behavior change. In Preventing AIDS (pp. 5-24). Boston, MA: Springer.

Rowley, J., Vander Hoorn, S., Korenromp, E., Low, N., Unemo, M., Abu-Raddad, L. J., et al. (2019). Global and regional estimates of the prevalence and incidence of four curable sexually transmitted infections in 2016. WHO Bulletin, June 2019.

Salazar, L. F., Crosby, R. A., DiClemente, R. J., Wingood, G. M., Lescano, C. M., Brown, L. K., et al. (2005). Self-esteem and theoretical mediators of safer sex among African American female adolescents: Implications for sexual risk reduction interventions. Health Education \& Behavior, 32(3), 413-427.

Satterwhite, C. L., Torrone, E., Meites, E., Dunne, E. F., Mahajan, R., Ocfemia, M. C. B., \& Weinstock, H. (2013). Sexually transmitted infections among US women and men: Prevalence and incidence estimates, 2008. Sexually transmitted diseases, 40(3), 187-193.

Schooler, D., \& Ward, L. M. (2006). Average Joes: Men's relationships with media, real bodies, and sexuality. Psychology of Men \& Masculinity, $7(1), 27$.

Schooler, D., Ward, L. M., Merriwether, A., \& Caruthers, A. S. (2005). Cycles of shame: Menstrual shame, body shame, and sexual decision-making. Journal of Sex Research, 42(4), 324-334.

Secor-Turner, M., Griese, E., Baete, D., Kenyon, D. B., \& Randall, B. A. (2015). Sexual risk behavior among frontier adolescents: Profiles of pregnancy risk and protection. Health Behavior and Policy Review, 2, 144-153.

Spitalnick, J. S., DiClemente, R. J., Wingood, G. M., Crosby, R. A., Milhausen, R. R., Sales, J. M., et al. (2007). Brief report: Sexual sensation seeking and its relationship to risky sexual behaviour among African-American adolescent females. Journal of adolescence, 30(1), 165-173.

Stefanile, C., Matera, C., Pisani, E., \& Zambrini, I. (2009). Insoddisfazione corporea in adolescenza: influenze di fattori bio-psicosociali. Psicologia della Salute, 2, 51-65.

Sterk, C. E., Klein, H., \& Elifson, K. W. (2004). Predictors of condom-related attitudes among at-risk women. Journal of Women's Health, 13(6), 676-688.

Strauss, T., \& von Maltitz, M. J. (2017). Generalising Ward's method for use with Manhattan distances. PloS one, 12(1), 1-21.

Tevendale, H. D., Lightfoot, M., \& Slocum, S. L. (2009). Individual and environmental protective factors for risky sexual behavior among homeless youth: An exploration of gender differences. AIDS and Behavior, 13(1), 154-164.

Thompson, J. K. (1990). Body image disturbance. New York: Pergamon Press.

Timmerman, G. M., \& Acton, G. J. (2001). The relationship between basic need satisfaction and emotional eating. Issues in Mental Health Nursing, 22(7), 691-701.

Vasilenko, S. A., Kugler, K. C., Butera, N. M., \& Lanza, S. T. (2015). Patterns of adolescent sexual behavior predicting young adult sexually transmitted infections: A latent class analysis approach. Archives of sexual behavior, 44(3), 705-715.

Voisin, D. R., Tan, K., \& DiClemente, R. J. (2013). A longitudinal examination of the relationship between sexual sensation seeking and STI-related risk factors among African American females. AIDS Education and Prevention, 25(2), 124-134.

Whitaker, D. J., \& Miller, K. S. (2000). Parent-adolescent discussions about sex and condoms: Impact on peer influences of sexual risk behavior. Journal of Adolescent Research, 15(2), 251-273.

Wingood, G. M., DiClemente, R. J., Harrington, K., \& Davies, S. L. (2002). Body image and African American females' sexual health. Journal of women's health \& gender-based medicine, 11(5), 433-439.

Youngblut, J. M., \& Casper, G. R. (1993). Focus on psychometrics single-item indicators in nursing research. Research in nursing \& health, 16(6), 459-465.

\section{Publisher's Note}

Springer Nature remains neutral with regard to jurisdictional claims in published maps and institutional affiliations. 